\title{
Envejecimiento demográfico y escenarios de vulnerabilidad urbana: ¿Hay siempre una relación conexa? Estudio de casos en las ciudades de Sevilla (España) y La Plata (Argentina)
}

\author{
Demographic aging and urban vulnerability scenes: Are they always connected? \\ Case study in the cities of Sevilla (Spain) and La Plata (Argentina)
}

\section{Carolina del Valle Ramos \\ cdvalle@us.es \\ Departamento de Geografía Humana. Universidad de Sevilla, España}

\section{María Eugenia Goicoechea \\ megoicoechea@yahoo.com.ar}

CONICET. Instituto Multidisciplinario de Historia y

Ciencias Humanas. Universidad de Buenos Aires.

Facultad de Arquitectura, Diseño y Urbanismo,

Argentina

Recepción: 17 Junio 2020

Aprobación: 08 Septiembre 2020

Publicación: 03 Mayo 2021

Cita sugerida: Valle Ramos, C. del y Goicoechea, M. E. (2021). Envejecimiento demográfico y escenarios de vulnerabilidad urbana: ¿Hay siempre una relación conexa? Estudio de casos en las ciudades de Sevilla (España) y La Plata (Argentina). Geograficando, 17(1), e089. https://doi.org/10.24215/2346898Xe089
Resumen: El envejecimiento poblacional se presenta como un fenómeno demográfico actual, consolidado en las ciudades tanto europeas como latinoamericanas. No obstante, los índices de envejecimiento se distribuyen de manera desigual al interior de estas, suponiendo un diálogo particular con las áreas de vulnerabilidad urbana. El objetivo del artículo es mostrar la evolución reciente del proceso de envejecimiento demográfico urbano y su desigual distribución socioespacial en las ciudades de Sevilla (España) y la Plata (Argentina), analizando, en perspectiva cuantitativa comparada, su relación con los escenarios de vulnerabilidad social y habitacional, ya que ello podría conllevar procesos de riesgo. La metodología utilizada es cuantitativa, centrada en análisis de fuentes estadísticas oficiales (censos, padrones, catastro). Los resultados obtenidos evidencian la importancia de considerar al envejecimiento demográfico como un estadio final del ciclo de vida del barrio, más que como condicionante en la vulnerabilidad urbana.

Palabras clave: Envejecimiento demográfico, Vulnerabilidad socio-habitacional, Sevilla, La Plata.

\begin{abstract}
The aging of population arises as a current demographic phenomenon, at present consolidated in both European and Latin American cities. However, aging rates are unevenly distributed across cities, in a particular dialogue with areas of urban vulnerability. The aim of the article is to show the recent evolution of the urban demographic aging process and its unequal socio-spatial distribution in the cities of Seville (Spain) and La Plata (Argentina), analyzing its relationship with scenarios of social vulnerability and housing in a comparative quantitative perspective, as it could result in processes of risk. The methodology used is quantitative, focused on analysis of official statistical sources (censuses, land registers, cadastre). The results obtained show the importance of considering demographic aging as an end stage of the neighborhood life cycle, rather than a condition for urban vulnerability.
\end{abstract}

Keywords: Demographic aging, Urban vulnerability risks, Seville, La Plata. 


\section{INTRODUCCIÓN}

El envejecimiento poblacional constituye un fenómeno demográfico que en la actualidad encontramos consolidado en el espacio urbano, tanto de países europeos como latinoamericanos. En las últimas décadas ha generado creciente interés para la vida en la ciudad, al punto de transformarse en un tema prioritario en las agendas de los organismos internacionales y nacionales. Naciones Unidas comenzó a abordarlos tempranamente a fines de los años sesenta del pasado siglo, y en 1982 en Viena celebró la primera Asamblea Mundial sobre envejecimiento, a partir de la cual se formuló el Plan de Acción Internacional, como primer instrumento de políticas y programas destinados a los adultos mayores. Desde ese momento, en los ámbitos urbanos se ha ido desarrollando toda una casuística de programas cuya orientación actual se dirige, en gran medida, a la búsqueda de lo que se ha terminado por denominar "ciudades amigables", término acuñado por la OMS para referirse a ámbitos urbanos que proporcionan a la población la posibilidad de desarrollar su derecho al "bien vivir". De hecho, el mismo organismo diseñó el proyecto Ciudades Amigables con Los Mayores, en el que se desarrollan algunos temas claves vinculados a la idea de trabajar para disminuir la vulnerabilidad socio habitacional de dicho colectivo; entre estos temas se encuentran, por ejemplo, la inclusión social, la participación cívica, los servicios comunitarios y de salud, la vivienda o la participación social, entre otros (Sugranyes y Mathivet, 2010). En el año 2010 se crea la Red Mundial de Ciudades y Comunidades Amigables con las Personas Mayores, y el 1 de octubre de 2020 está previsto que entre en vigor la denominada Década del Envejecimiento Saludable (2021-2030). Los esfuerzos de la OMS estarán centrados en trabajar para mejorar la vida de las personas mayores, sus familias y las comunidades en las que viven, con gran atención a los ámbitos urbanos (CEPAL, 2002).

El envejecimiento demográfico es un fenómeno que frecuentemente va ligado al de vulnerabilidad socio habitacional. Aunque desde un punto de vista biológico debe ser entendido como logro de las sociedades desarrolladas en lo que a incremento de la esperanza de vida se refiere, lo cierto es que, desde un punto de vista social, una gestión inadecuada de dicho proceso genera indiscutiblemente estadios de vulnerabilidad social. Hechos como la dependencia, los problemas sanitarios o las deficiencias en las viviendas y la falta de accesibilidad espacial son algunos de los factores que incrementan el grado de vulnerabilidad de un espacio, y también son algunos de los que se ocupan en la actualidad los estudios tanto de Gerontología Urbana, como el Urbanismo Gerontológico (Sánchez, 2007). En gran medida los análisis que se vienen realizando sobre envejecimiento demográfico y vulnerabilidad urbana ponen de relieve la existencia de un vínculo entre ambos procesos, especialmente relacionados en la teórica de la amenaza y el riesgo, tal y como explica Osorio Pérez (2017). El colectivo de población mayor presenta menores posibilidades para hacer frente a esos riesgos, lo que suele derivar en escenarios de pobreza, desigualdad y desventaja.

Así pues, la investigación parte de la hipótesis de que en los ámbitos urbanos el proceso de envejecimiento demográfico imprime a dichos espacios situaciones de riesgo que incrementan la vulnerabilidad socio habitacional. Para corroborar dicha hipótesis se ha recurrido al estudio de casos. Concretamente, para ello se ha analizado la evolución reciente del proceso de envejecimiento demográfico urbano, y su desigual distribución socioespacial, en las ciudades de Sevilla (España) y la Plata (Argentina), identificando los sectores más envejecidos, analizando los posibles escenarios de vulnerabilidad social y habitacional, y comprobando la existencia de procesos de riesgo (pobreza, desigualdad y desventajas) en dichos sectores. Con ello se evidencia la importancia que tienen en la génesis de los escenarios de vulnerabilidad urbana, los llamados "ciclos de vida de los barrios", y la importancia de realizar en ellos medidas de rehabilitación y regeneración en viviendas y edificios, que minimicen los efectos (riesgos) derivados del envejecimiento demográfico y mejoren la experiencia del habitar para este grupo sin poner en peligro su permanencia en estos.

La elección de estas ciudades como casos de estudio responde a que ambas son sede político-administrativa de importantes jurisdicciones subnacionales: La Plata de la provincia de Buenos Aires y Sevilla de la región de Andalucía. 
Ambas ciudades conforman áreas metropolitanas con similar estructura poblacional, aunque representan diferente jerarquía dentro del sistema urbano. Desde el punto de vista de las conformaciones urbanas, ambas ciudades - con intensidad variable en conformidad con los ritmos económicos coyunturales de auge y crisishan visto expandir sus áreas metropolitanas, sobre todo en las dos últimas décadas, como consecuencia de los mecanismos del mercado inmobiliario (Coq Huelva, 2012), el cual, en el caso de La Plata, se desarrolló mediante la tipología de urbanizaciones cerradas y, también, por procesos de producción social del hábitat (Relli, 2018; Frediani, 2009). El encarecimiento del valor del suelo en las áreas centrales implicó la expulsión de población autóctona hacia barrios periféricos, lo que provocó la pérdida de tejido residencial, especialmente de familias jóvenes. Aun cuando acogen un elevado número de comercios, sedes institucionales y servicios, las zonas centrales de ambas ciudades registran un estancamiento del número de residentes, e incluso en algunos momentos pérdidas demográficas. A ello se le añaden procesos de gentrificación (Díaz Parra, 2009) y turistificación de sus centros históricos (más marcados para el caso sevillano). Finalmente, desde el punto de vista de los procesos de vulnerabilidad urbana, ambas ciudades registran barrios vulnerables en los que la población residente sufre claros efectos de desfavorecimiento y desigualdad (Valle Ramos, Egea Jiménez y Nieto Calmaestra, 2020).

Ante las premisas anteriores, el objetivo de esta investigación asienta sus bases en la necesidad de conocer el impacto que imprime el envejecimiento demográfico al tejido urbano, en lo que a vulnerabilidad socio habitacional se refiere, desde una perspectiva comparada entre Sevilla y La Plata. Ello implica considerar las características que se observan en el grupo social de los adultos mayores en cada contexto, y su relación con el espacio urbano en el que se asientan (reconociendo la desigualdad territorial), así como también, el diálogo que establecen con las transformaciones socio habitacionales que sus barrios atraviesan. En adición a esta mirada comparada entre Sevilla y La Plata se identificaron en ambas ciudades áreas pericentrales con alto grado de envejecimiento demográfico; se partió de los valores medios de cada barrio, y, a continuación, se avanzó en un análisis más detallado. Los ámbitos elegidos, cuyas localizaciones pueden observarse en la figura 1, han sido las barriadas de Rochelambert, en el distrito Cerro Amate y San Pablo C, en el distrito San Pablo (en Sevilla), y entorno Monasterio en la localidad de Villa Elvira y entorno Estación en Tolosa (en La Plata).

\section{FIGURA 1}

Localización de los ámbitos de estudio: las ciudades de Sevilla (España) y La Plata (Argentina) y los casos de estudio
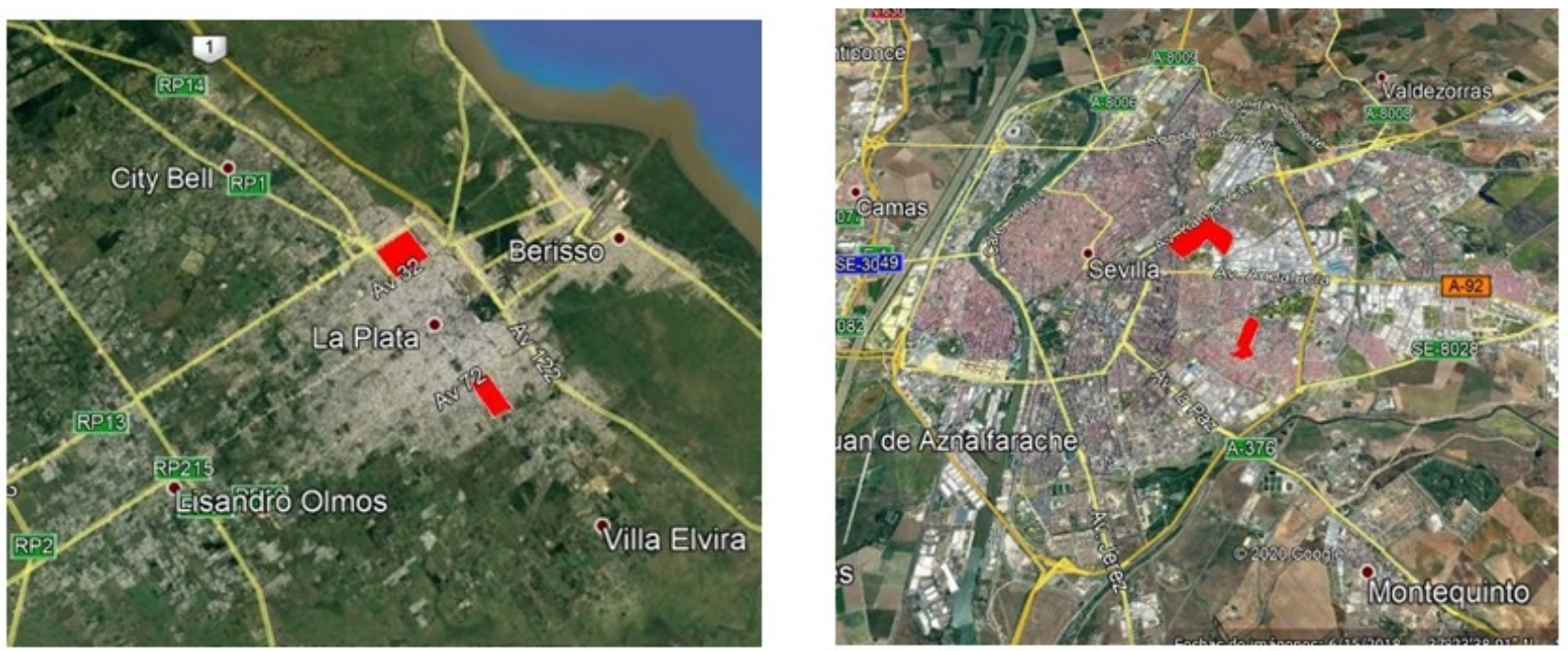


\section{ENFOQUE TEÓRICO METODOLÓGICO: ENVEJECIMIENTO POBLACIONAL Y VULNERABILIDAD SOCIODEMOGRÁFICA EN LAS CIUDADES}

El envejecimiento demográfico alude a un proceso de cambio en la composición estructural de una población, por el cual el peso relativo de los grupos etarios superiores (comúnmente delimitada como mayores de 64 años) supera ampliamente el valor del $7 \%$-umbral que ha establecido un indicador de procesos de envejecimiento-, en detrimento de los otros grupos de edad. Responde en mayor medida a dos fenómenos concomitantes vinculados a la mejora en la calidad de vida, con el consecuente aumento en la esperanza de vida de la población, y al descenso de la fecundidad (Tomas, 2007). Genera efectos muy complejos y diversos para la vida en sociedad, ya que implica la emergencia de un nuevo estrato social y demográfico con demandas, necesidades, responsabilidades y capacidades propias de un grupo poblacional específico.

En cuanto a sus implicancias para la vida social, desde los enfoques sociológicos y antropológicos sobre el envejecimiento poblacional se reconocen los esfuerzos por comprender las estrategias de reproducción de este grupo social, que, por su parte, asume roles, formas de actuar y pensar específicas. Particularmente en España, en la década del ochenta tomaron protagonismo los abordajes etnográficos sobre la vejez, desde las teorías de la marginación (o la desconexión), así como también los abordajes sobre el desafío que supone este fenómeno para la política pública española (Rodríguez Rodríguez, 2018, Casado, 2000, Abades Porcel y Rayón Valpuesta, 2012). En el campo de la demografía también se reconocen aportes (Pérez Díaz, 1998, Pérez Díaz y Abellán García, 2018). En Argentina, por su parte, se reconocen estudios sobre la temática para ciudades específicas, como Bahía Blanca (Prieto y Formiga, 2008), Córdoba (Peláez, 2004, 2006; Peláez y Molinatti, 2016 y Acosta, 2019) y Mar del Plata, que, por su infraestructura sanitaria y su paisaje costero, en las últimas décadas se ha convertido en un destino residencial privilegiado entre los adultos mayores del país (Laterza Calosso, 2015).

Entre los enfoques sociológicos, el envejecimiento resulta también una dimensión importante en la dinámica de la desigualdad y se aborda desde la perspectiva de la vulnerabilidad social (Moser, 1998; Kaztman, 1999; Saraví, 2006). Estos trabajos reconocen variaciones en el nivel de vulnerabilidad social de los hogares en función de su capacidad (definida a partir de la noción de activos) para adaptarse y responder a los cambios en el contexto, de manera tal de aprovechar la estructura de oportunidades que este ofrece y reducir la exposición a riesgos frente a eventos potencialmente adversos. La vejez, en este sentido, es considerada un condicionante o factor de riesgo, que incide en la calidad de vida y las capacidades de los grupos poblacionales. Comprende un aspecto de la vulnerabilidad demográfica, componente de la desventaja social que limita la acumulación de recursos (Rodríguez Vignoli, 2000), pero, al mismo tiempo, promueve una dinámica particular y específica en la vulnerabilidad social del hogar. Por ejemplo, el nivel de vida de un hogar a partir de la condición de actividad de sus miembros se ve afectado por la relación de dependencia resultante. La presencia de adultos mayores que representan población inactiva imprime una desventaja social con relación a otros hogares con mayor proporción de miembros económicamente activos. No obstante, esto también puede ser un beneficio cuando el adul to mayor percibe jubilaciones y/o pensiones. Asimismo, la cohabitación reduce la condición de vulnerabilidad de la población envejecida, ya que resuelve de manera informal los problemas de aislamiento y de asistencia gerontológica (Sánchez González, 2007; Peláez y Molinatti, 2016).

Por último, a partir del análisis de esta asociación entre vulnerabilidad social y envejecimiento demográfico con emplazamiento en las ciudades, se incorpora un tercer concepto, que aporta nuevas aristas y complejidad al análisis. Refiere al concepto de vulnerabilidad urbana, entendido como un proceso de malestar en las ciudades producido por la combinación de múltiples dimensiones de desventaja, que incide tanto sobre las posibilidades de movilidad social ascendente, como de superación de condiciones sociales de exclusión o marginación social. Quedan incluidas aquíla dimensión sociodemográfica (en donde se reconoce al envejecimiento poblacional), la socioeconómica, la residencial y la subjetiva (Alguacil, Camacho, y Hernández, 2014). 
Esta mirada a la vulnerabilidad urbana, entonces, lleva a dejar de pensar el territorio como un mero contenedor de las características sociales de quienes lo habitan, y a incorporar al análisis la trayectoria de los propios barrios (proceso de urbanización, mercado inmobiliario, estructura urbana), la cual también incide fuertemente en la dinámica del impacto desigual provocado por las desventajas sociales y el envejecimiento (Egea Jiménez y Nieto Calmaestra, 2015). En esta línea pueden pensarse adicionalmente las relaciones entre el envejecimiento demográfico y el "ciclo de vida" de los barrios dentro de la ciudad. Antecedentes de investigación al respecto (Peláez y Molinatti, 2016) señalan una tendencia a la concentración de mayores niveles de adultos mayores en las áreas centrales o más consolidadas de la ciudad, aunque en la agenda académica aún resta comprender cómo afectan particularmente a este grupo poblacional los procesos de renovación de las áreas centrales, cuáles son sus estrategias de reproducción y cómo afrontan los cambios en las dinámicas urbanas que acompañan a estas transformaciones socio territoriales (verticalización, aumento de las actividades terciarias, aumento del costo de vida, pérdida de identidad barrial).

En consonancia con los antecedentes y perspectivas de estudio esbozados en relación al problema del envejecimiento demográfico en las ciudades y su diálogo con la vulnerabilidad urbana, el diseño metodológico de este artículo contempla la medición del envejecimiento demográfico a partir de: el porcentaje de mayores de 64 años, que es el umbral internacionalmente reconocido en las investigaciones de poblaciones desarrolladas; el índice de envejecimiento -cociente entre la población mayor de 64 años y la población menor de 16 años, expresado en porcentaje-, que facilita la valoración de la existencia de más o menos población dependiente en un territorio; la edad media de la población; el índice de sobre- envejecimiento -cociente entre la población mayor de 80 años con respecto a la de más de 64 años-, que sirve para conocer la intensidad del fenómeno, y, finalmente, la tasa de dependencia, que relaciona el envejecimiento con la actividad.

En cuanto al nivel de vulnerabilidad social y urbana de los barrios, se han utilizado como variables el porcentaje de población desempleada, la tasa de analfabetismo, el porcentaje de población extranjera con respecto al total de población del ámbito (indicador de presión migratoria), la renta media de los hogares y el tamaño de las viviendas. Se trata de variables "síntomáticas", que permiten valorar el grado de vulnerabilidad socio habitacional de las barriadas en estudio. La elección de las mismas tiene el propósito de alcanzar una caracterización general en cada caso de estudio, y, a partir de ello, establecer una mirada comparativa. Responde, por un lado, a las limitaciones propias de la disponibilidad del dato estadístico, y, por el otro, a una consideración de las particularidades históricas, macroeconómicas y geográficas de los países en los cuales estas urbes se localizan.

Las fuentes empleadas han sido datos estadísticos oficiales proporcionados por los últimos censos de población de España (2011) y Argentina (2010), y datos catastrales. Estas son las únicas fuentes que con una desactualización de diez años- proporcionan información demográfica y socio habitacional para todas las unidades censales, aunque los resultados se han analizado desde la escala geográfica del barrio. El estudio presenta tres fases: a) identificación de los barrios con alto grado de envejecimiento demográfico en ambas ciudades de estudio y análisis de la evolución del proceso en cada uno; b) observación en los barrios envejecidos de los niveles de vulnerabilidad socio habitacional que presentan, y, c) evaluación del grado relacional entre los procesos de envejecimiento demográfico, la existencia de desigualdades socio habitacionales y sus condicionantes con respecto a escenarios de desventajas. 


\section{RESULTADOS Y DISCUSióN}

\subsection{Envejecimiento demográfico en Sevilla y La Plata: procesos convergentes y barrios envejecidos}

El crecimiento demográfico que han tenido las ciudades de Sevilla y la Plata presenta tendencias distintas. Desde 1981 a la actualidad, La Plata ha seguido un crecimiento ascendente interrumpido solo a principios de la década de los noventa, pero que no duró más de 5 o 6 años. Ha pasado, por tanto, de registrar un total de 564.847 residentes en 1981 a 687.378 en el 2015. La ciudad de Sevilla, por el contrario, presenta una tendencia de estabilización asociada desde hace una década a una pérdida de carga demográfica. Así pues, de tener en 1981 un total de 653.833 habitantes ha pasado a 688.711, valor que, según las proyecciones realizadas para el año 2025, continuaría descendiendo. Por tanto, aunque hoy día ambas ciudades registran volúmenes demográficos muy parecidos, lo cierto que es que las tendencias que explican los valores actuales han sido diferentes.

Con respecto a la distribución espacial de la población, en La Plata el número de residentes es mayor en los centros comunales situados al norte -San Carlos, Melchor Romero, Gonnet, Gorina y Ringuelet-, frente a los de la zona sur -Etcheverry, Los Hornos y Villa Elvira-, que registran los menores volúmenes de población. En Sevilla, son los distritos Este, Cerro-Amate y Macarena los que más volumen de población aportan al conjunto de la ciudad, frente a Los Remedios, Bellavista-La Palmera y Triana, que registran menos de un 7 $\%$ cada uno del total de habitantes de la ciudad. Con respecto a las densidades medias de población, Sevilla registra un valor de 4.896,55 hab $/ \mathrm{km}^{2}$, mientras que en la Plata la media es de 585,2 hab $/ \mathrm{km}^{2}$. En este sentido, también existen diferencias zonales. Así, por ejemplo, el centro comunal platense con el valor más alto es el Casco Urbano, con una densidad superior a 16.400 personas por $\mathrm{km} 2$, volumen que se expande por los radios censales que lo circundan, donde las densidades llegan en esos a casos a valores en torno a los 6.000 residentes por km2. En Sevilla, las mayores densidades las registran los distritos del Casco Antiguo, Macarena y Nervión, frente a Los Remedios y Bellavista, que obtienen las menores densidades.

Con respecto al envejecimiento demográfico, las ciudades tanto europeas como latinoamericanas presentan situaciones de envejecimiento que se corresponden con sus estadios con respecto a la transición demográfica. España y Argentina son muestra de ello. Las ciudades españolas registran niveles de envejecimiento alto, especialmente aquellas que son capitales de provincia de tamaño medio. La ciudad de Sevilla ha incrementado su porcentaje de población mayor de 64 años en la última década, y presenta una estructura de población de sociedades envejecidas. Del boom demográfico de los años 80 del siglo XX, la ciudad ha llegado en la actualidad a que un $18,76 \%$ de su población total supere el umbral de tercera edad. De hecho, la edad media de la población se sitúa en los 42,65 años. Su índice de envejecimiento en 2019 es de 116,86\%, mientras que en 2000 era de 97,6\%, y en 2004 de 104,6\% (Valle Ramos, 2007). Su índice de sobre envejecimiento es de $31,65 \%$ y la tasa de dependencia se sitúa en un valor de 53,43\%. Por barrios, como queda recogido en la figura 2, la mayor parte de los que registran altos índices de envejecimiento (valores superiores a 212) se localiza en los distritos de Triana (el 24,80\% de los residentes superan los 64 años), Macarena (con un 21,3\% de su población) y San Pablo (con un $23 \%$ ), aunque también encontramos en Nervión (20,8 \%) y Los Remedios (20,3\%).

La mayor parte de las ciudades de Argentina se ha sumado al proceso de envejecimiento algo después que las españolas, consecuencia del retraso en los procesos demográficos que condicionan el comportamiento de las variables explicativas de la transición demográfica. La ciudad de la Plata registra un porcentaje de población de tercera edad algo menor que la ciudad de Sevilla, con un valor de un $12 \%$ del total de residentes, frente al $23 \%$ que tiene menos de 15 años. La presencia de la Universidad Nacional de La Plata imprime una vida urbana universitaria que incrementa el peso de la población joven y tiene impactos demográficos significativos 
sobre la tendencia al envejecimiento. No obstante, es también considerada una ciudad envejecida, en tanto supera el parámetro del $7 \%$ establecido por UN, y participa, al igual que Sevilla, del proyecto global Ciudades Amigables con la Edad de la ONU (UN, 2015). Los valores más altos los registran el Casco Histórico, Tolosa, City Bell y Ringuelet, todos ellos con porcentajes en torno al $12 \%$. En el lado opuesto se encuentran las localidades de Melchor Romero, Lisandro Olmos, Hernández, Arturo Seguí y Gorina, donde el porcentaje de población envejecida no supera el $6 \%$.

FIGURA 2

Porcentaje de población mayor de 64 años según radio o sección censal. Sevilla, 2011 y La Plata, 2010.
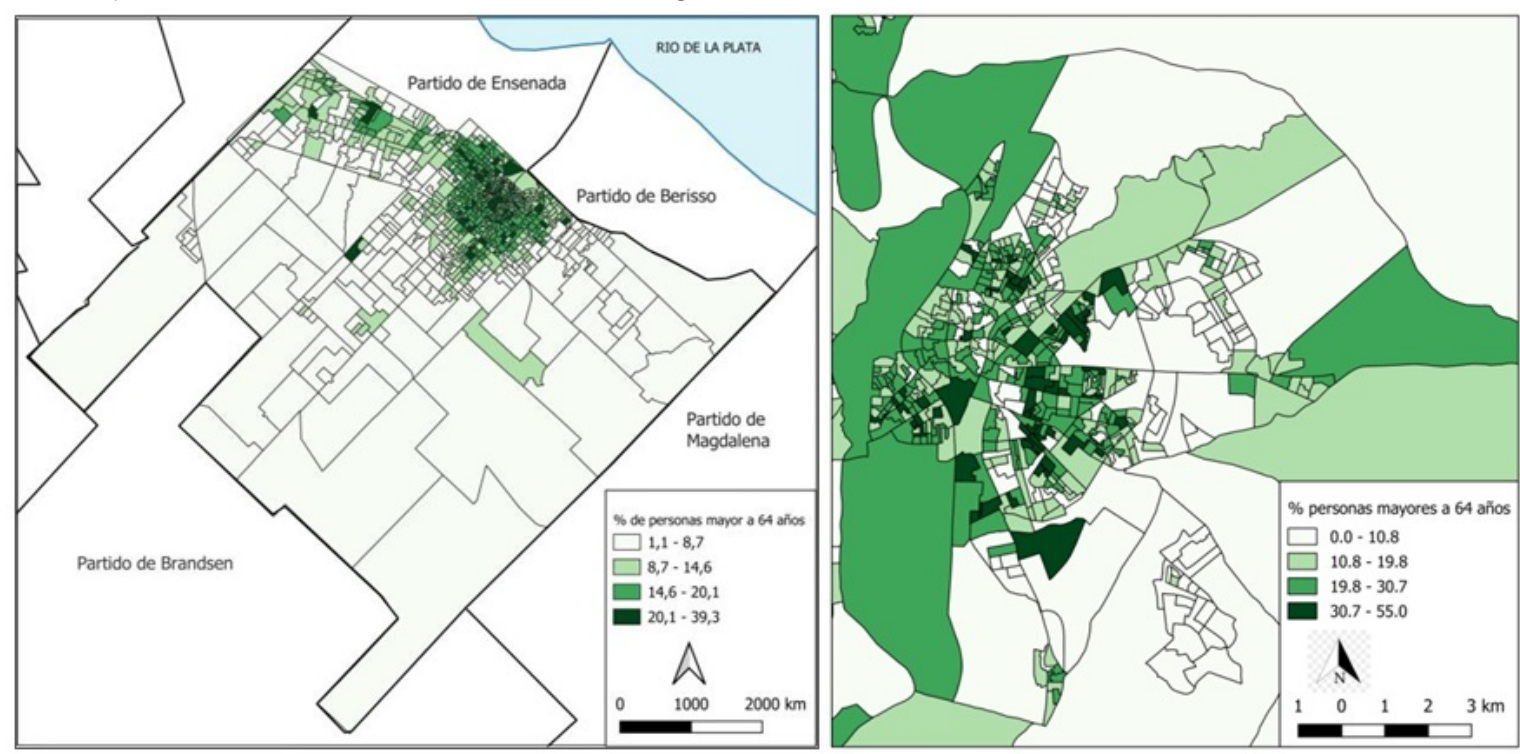

Fuente: elaboración propia a partir de los datos censales del INE (2011) e INDEC (2010).

Nota: intervalos dispuestos en base a los cortes naturales de la distribución en cada caso.

Por tanto, si comparamos cómo se distribuyen los procesos de envejecimiento demográfico en la estructura interna de ambas ciudades en función de la conformación urbana de las mismas, se comprueba en ambos casos la influencia del ciclo de vida de los barrios. Aquellos barrios y centros comunales que iniciaron antes su colmatación urbanística acogen los porcentajes más altos de población envejecida, mientras que aquellos en los que el planeamiento se ha configurado de forma más reciente, aún presentan índices menos elevados de vejez y dependencia. Sin embargo, hay que destacar que envejecimiento y pobreza no se relacionan de manera simple y lineal. Es por ello por lo que se hace interesante -y lo hacemos en el siguiente epígrafe- observar los niveles de vulnerabilidad socio habitacional que tienen las zonas más envejecidas, reconocidas en los casos de Rochelambert (Cerro Amate) y San Pablo C (San Pablo) en Sevilla, y entorno Monasterio (Villa Elvira) y entorno Estación Tolosa (Tolosa) en La Plata.

Se tomaron ciertas consideraciones metodológicas a fin de facilitar la lectura comparada de estos fenómenos emplazados en contextos diferentes. Refieren todas a áreas peri centrales en las que habita población asentada hace varias décadas con tradición obrera, y bajo un régimen de tenencia de la vivienda y el suelo formal. Tanto en el caso de La Plata como en Sevilla se ha optado por tomar sectores de localidades y/o barrios que contemplan un envejecimiento poblacional elevado y características socio habitacionales homogéneas en su interior. 


\subsection{El "ciclo de vida los barrios" en el análisis de la vulnerabilidad urbana y socio habitacional}

Para la ciudad de Sevilla se han analizado cinco indicadores de envejecimiento demográfico de los barrios de Rochelambert y San Pablo C. Como puede apreciarse en la figura 3, en ambos ámbitos el porcentaje de población envejecida es alto, y supera tanto la media de la ciudad de Sevilla (18,76 \%) como la media de sus distritos (17,85 \% en Cerro Amate y 23,10 \% en San Pablo-Santa Justa). Así, en Rochelambert nos encontramos que el $24,74 \%$ de sus habitantes superan los 65 años de edad, con un índice de envejecimiento de 174,60, una edad media de 47,75 años, un índice de sobre envejecimiento de 47,03\% y una tasa de dependencia de 63,68 \%. En el barrio de San Pablo C el porcentaje de mayores de 64 años asciende a un 28,46 $\%$ de su población, con un índice de envejecimiento de 240,89, una edad media de 44,47 años, un índice de sobre envejecimiento del $46,19 \%$ y un valor de $67,43 \%$ en la tasa de dependencia.

En La Plata, aunque con menor intensidad respecto al caso sevillano, el peso relativo de la población envejecida en Tolosa norte (17,34\%) y Monasterio (15,39\%) también es mayor que el promedio de la ciudad $(11,28 \%)$. El índice de envejecimiento platense $(50,98)$ también es significativamente menor, aunque destaca aquí la disimilitud geográfica en la que se distribuye, destacándose el sector de Tolosa, que observa un valor de 98,66. Finalmente, se destaca una particularidad en el caso de Monasterio, que, con una media de edad e índice de envejecimiento superiores al promedio platense, observa un índice de sobre envejecimiento y una tasa de dependencia inferiores.

Como hemos comprobado, los índices de dependencia demográfica entre ambas ciudades son similares, aunque por motivos diferentes: en Sevilla, a razón del mayor peso en los estratos +64 años; en La Plata, de los de -14 años.

FIGURA 3

Indicadores de envejecimiento demográfico en los ámbitos de estudio

\begin{tabular}{|c|c|c|c|c|c|c|}
\hline & $\begin{array}{c}\text { Barrio de } \\
\text { Rochelam } \\
\text { bert } \\
\text { (Sevilla) }\end{array}$ & $\begin{array}{c}\text { Barrio de San } \\
\text { Pablo C } \\
\text { (Sevilla) }\end{array}$ & $\begin{array}{c}\text { Ciudad de } \\
\text { Sevilla }\end{array}$ & $\begin{array}{l}\text { Tolosa- } \\
\text { estación } \\
\text { (La Plata) }\end{array}$ & $\begin{array}{r}\text { Monasterio } \\
\text { (La Plata) }\end{array}$ & $\begin{array}{c}\text { Ciudad de La } \\
\text { Plata }\end{array}$ \\
\hline $\begin{array}{c}\text { Población total } \\
\text { (personas) }\end{array}$ & 6.670 & 3.809 & 688.711 & 15.340 & 7.761 & 654.324 \\
\hline Extensión $\left(\mathrm{Km}^{2}\right)$ & 0,20 & 0,63 & 140,8 & 2,38 & 1,38 & 926 \\
\hline $\begin{array}{c}\% \text { de mayores de } \\
64 \text { años }\end{array}$ & 24,74 & 28,46 & 18,76 & 17,34 & 15,39 & 11,28 \\
\hline $\begin{array}{l}\text { Edad media } \\
\text { (años) }\end{array}$ & 44,47 & 47,74 & 42,65 & 39 & 37 & 34 \\
\hline $\begin{array}{c}\text { In dice de } \\
\text { envejecimiento }\end{array}$ & 174,60 & 240,89 & 116,86 & 98,66 & 81,45 & 50,98 \\
\hline $\begin{array}{l}\text { Îndice de sobre } \\
\text { envejecimiento }\end{array}$ & 46,19 & 47,03 & 31,65 & 25,06 & 16,31 & 23,02 \\
\hline $\begin{array}{c}\text { Tasa de } \\
\text { dependencia }\end{array}$ & 63,68 & 67,43 & 53,43 & 53,67 & 41,90 & 50,20 \\
\hline
\end{tabular}

Fuente: elaboración propia a partir de los datos de Padrón continuo del INE (2019) e INDEC (2010).

El análisis sobre la evolución que ha tenido el envejecimiento demográfico desde 1981 a la actualidad en las dos barriadas sevillanas confirma que ambos sectores comenzaron el proceso a principios de la década de los 90 del pasado siglo, y se acentuó entre el 2011 y el 2019 (Figura 4). El comportamiento de los porcentajes 
en los sectores de La Plata es idéntico que el observado en Sevilla, aunque entre el 2001 y 2010 el ritmo de crecimiento parece haberse estabilizado.

FIGURA 4

Evolución de la población mayor de 64 años en los sectores de estudio

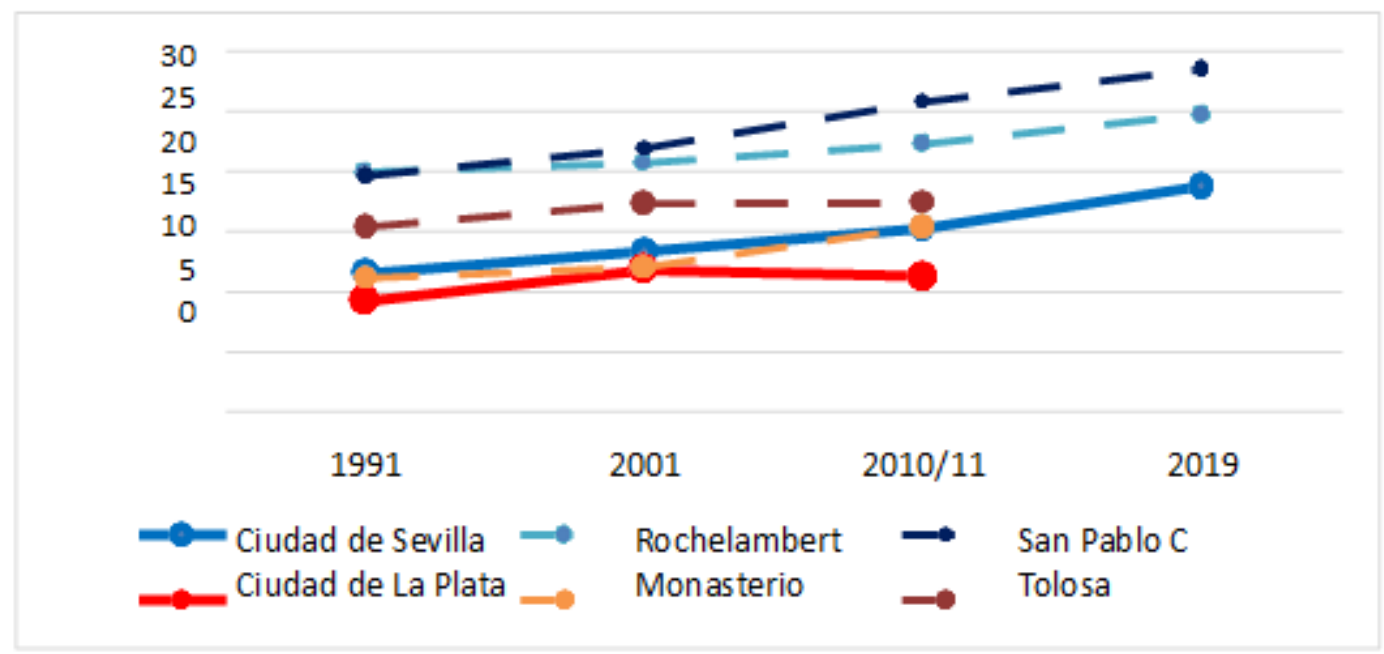

Fuente: elaboración propia a partir de los datos de Padrón continuo del INE (2019) e INDEC (2010).

El barrio de Rochelambert, que forma parte de la segunda corona urbana de la ciudad de Sevilla, empieza a construirse en los años 50 del siglo XX. Era un arrabal de la ciudad que fue colmatando su superficie urbanizada con edificaciones hasta la década de los años 70. Combina, como se observa en la figura 5, la existencia de autoconstrucciones de 1 y 2 plantas con edificios de hasta 15 alturas. El barrio de San Pablo C tiene su origen en los años 60 del siglo XX. Su construcción está ligada a la Obra Sindical del Hogar y su origen, al realojo de población de, por una parte, otros barrios suburbanos que fueron afectados por la Riada del Tamarguillo de 1961, y, por otra, de inmigrantes que llegaron a Sevilla a raíz de su desarrollo industrial. Ambos barrios han colmatado su espacio urbano y han registrado durante los últimos años actuaciones de rehabilitación y regeneración urbana.

En La Plata, Tolosa tiene su origen en la inauguración de la estación homónima en 1983, tras la extensión del ferrocarril en conexión con la ciudad de Buenos Aires. Tiene tradición de inmigración italiana y obrera vinculada a la industria automotriz de trenes, dado que alberga a los talleres ferroviarios más grandes del país, inaugurados en 1887 (Vallejo, 2000). Por su parte, el poblamiento de Monasterio es más tardío. Su formación data de 1970 en los bañados de Monasterio, cuando inicia la construcción de un complejo habitacional de alrededor de 1000 viviendas para trabajadores del Astillero Río Santiago y personal de la Marina, entre otras dependencias de militares. Estas obras, finalizadas en 1986, motorizaron la apertura de calles, extensión de servicios urbanos y dotación de equipamientos educativos y de salud. 
FIGURA 5

Imágenes de los casos de estudio. Entorno y tipología edilicia

Tolosa (La Plata)

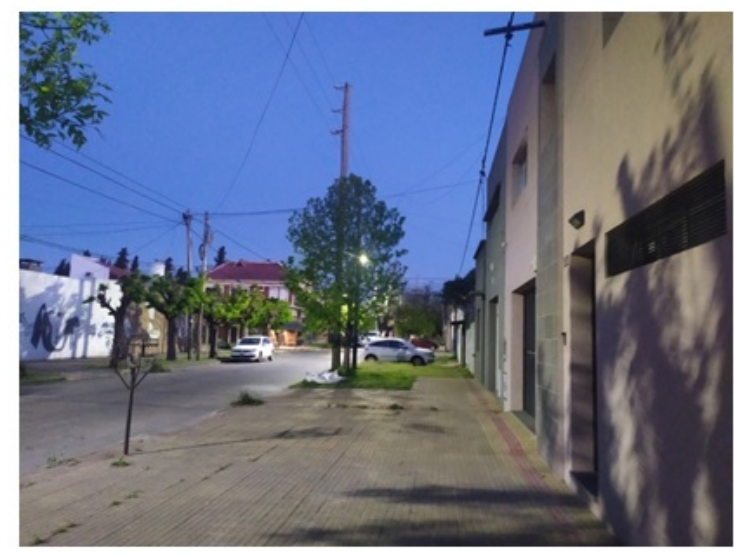

Rochelambert (Sevilla)
Monasterio (La Plata)

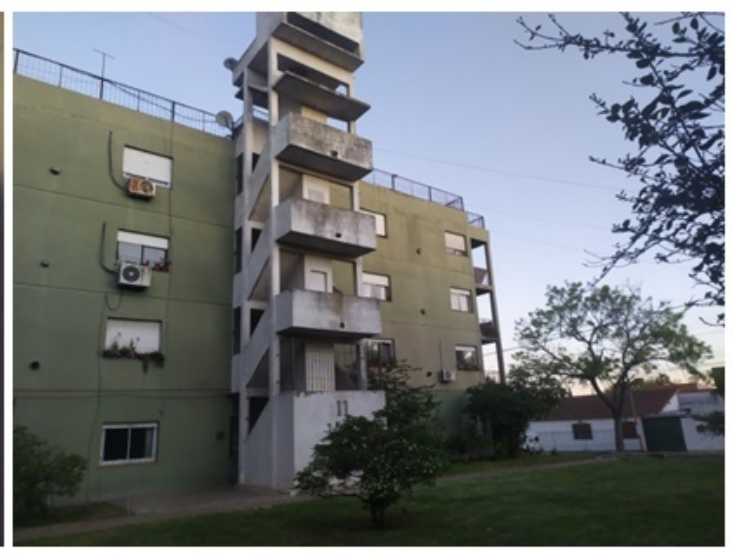

San Pablo C. (Sevilla)

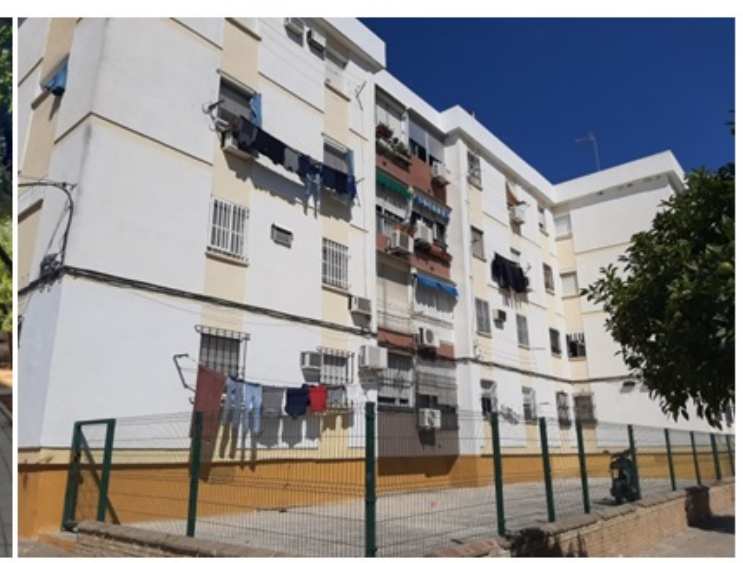

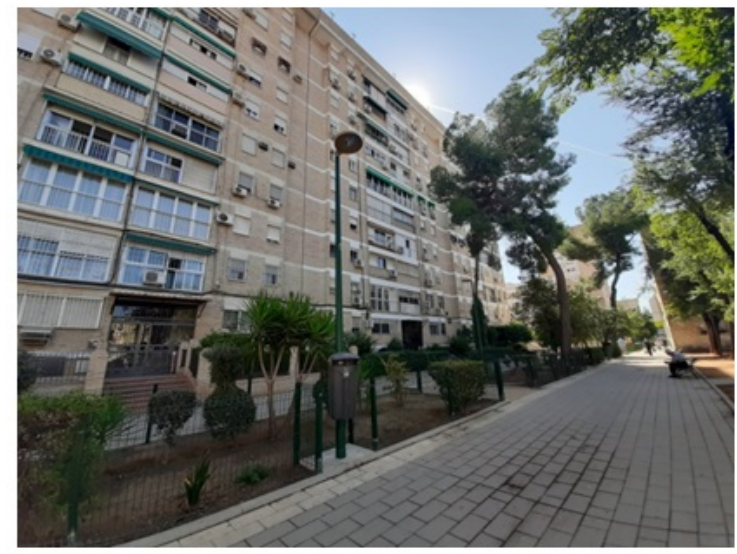

Fuente: Fotografías tomadas Valle Ramos y Goicochea (octubre, 2020)

Desde el punto de vista de las vulnerabilidades (sociodemográfica y habitacional), que han sido analizadas a partir de las variables sintomáticas que aparecen en la figura 6, en el barrio de San Pablo C destaca una alta tasa de paro (desocupación) de su población $(24,8 \%$ ) a lo que se une una baja renta (ingreso) per cápita de sus habitantes (7.845 euros). Además, son numerosos los hogares unipersonales de mayores de 65 años que cuentan con bajas pensiones. Desde la perspectiva habitacional, se trata en su mayoría de un parque de viviendas antiguas (en torno a 50 años), que tienen una superficie media de $70 \mathrm{~m} 2$ y cuyo precio de mercado es bajo con respecto a la media de la ciudad. Por su parte, el barrio de Rochelambert acoge también a un número importante de hogares de población mayor de 64 años (19,1 \%). Sin embargo, es destacable la presión migratoria del mismo $(6,8 \%)$, que supera el valor medio de Sevilla. La tasa de paro es alta y la renta per cápita por habitante tan solo se sitúa de media en unos 8.081 euros. El parque de viviendas supera los 60 años de antigüedad, y aunque el tamaño de las viviendas está en torno a la media de la capital, el precio medio del $\mathrm{m} 2$ se sitúa muy por debajo de la misma (1.066 euros). Por tanto, en los dos barrios de estudio de la ciudad de Sevilla, la vulnerabilidad debe ser entendida más desde la variable económica que desde la habitacional, y ello se ve influido por el alto porcentaje de población envejecida, cuyos niveles de renta son bajos a consecuencia de su inactividad. Sin embargo, y según el Informe anual 2019 sobre las Condiciones de Vida en las ciudades europeas (proyecto Urban Audit), ninguno de los barrios de estudio se encuentra entre los 15 con menor renta media anual por habitante. Y tampoco son clasificados comoáreas de alta vulnerabilidad en el Atlas de la Vulnerabilidad Urbana de España (2011). Por tanto, podemos afirmar que en estos barrios el envejecimiento 
demográfico es una variable más a tener en cuenta con respecto al último estadio del propio ciclo de vida de los mismos; una situación que puede agravar ciertos aspectos de desventaja social, pero que no tiene por qué conllevar de forma directa a la existencia de pobreza y/o marginalidad.

Esta última reflexión también es pertinente para pensar la situación en la ciudad de La Plata, en tanto las áreas seleccionadas, con marcado envejecimiento poblacional, no son las que observan los mayores niveles de vulnerabilidad social. Contemplan niveles relativamente bajos en cuanto a desocupación y analfabetismo: Tolosa, 3,12 y 4,02 \% y Monasterio, 3,68 y 4,11\%, respectivamente. No se dispone de datos actualizados y desagregados sobre el nivel de ingresos, siendo que la única fuente que lo provee -Encuesta Permanente de Hogares- solo es representativa a nivel del aglomerado urbano Gran La Plata. No obstante, una aproximación a ello desde el indicador censal de Necesidades Básicas Insatisfechas (NBI) ${ }^{1}$ confirma que las condiciones socio habitacionales son relativamente mejores. El promedio de hogares con al menos un componente de NBI es de 8,42 \% para el total de la ciudad, mientras que en Tolosa es del 0,66 \% y en Monasterio, del 1,94 $\%$. Inversamente al caso sevillano, los componentes habitacionales -hacinamiento y vivienda deficitariason los que tienen mayor incidencia en la vulnerabilidad los hogares platenses, tanto para el promedio de la ciudad como para los barrios alcanzados. Tolosa observa un comportamiento similar a la media de la ciudad en cuanto a presencia de hogares unipersonales habitados por mayores de 64 años, mientras que en monasterio es bajo y apenas supera el $3 \%$. Lo mismo acurre con el tamaño y valor del $\mathrm{m} 2$ de las viviendas. Nos obstante, ambas localidades presentan una fuerte heterogeneidad socio habitacional interna, en tanto los datos agregados a nivel de localidad deben tomarse con reservas para pensar los sectores bajo análisis. Para el caso de Monasterio, Villa Elvira combina suelo rural y urbano, atributo que distorsiona los valores de suelo de las viviendas emplazadas. a partir de estos datos de Matteucci (2015), en ambos sectores se observan dinámicas de valorización inmobiliaria pero que no responden a una mayor demanda agregada de espacio residencial, ya que en los últimos 20 años registran niveles estables de crecimiento demográfico. 
FIGURA 6

Variables sintomáticas de vulnerabilidad socioeconómica y habitacional en los sectores estudiados

\begin{tabular}{|c|c|c|c|c|c|c|}
\hline & $\begin{array}{c}\text { Barrio de } \\
\text { Rochelam b } \\
\text { ert } \\
\text { (Sevilla) }\end{array}$ & $\begin{array}{l}\text { Barrio de } \\
\text { San Pablo } \\
\text { C (Sevilla) }\end{array}$ & $\begin{array}{l}\text { Ciudad de } \\
\text { Sevilla }\end{array}$ & $\begin{array}{c}\text { Tolosa norte } \\
\text { (La Plata) }\end{array}$ & $\begin{array}{c}\text { Monasteri } \\
\circ(\mathrm{La} \\
\text { Plata) }\end{array}$ & $\begin{array}{l}\text { Ciudad de } \\
\text { La Plata }\end{array}$ \\
\hline $\begin{array}{l}\% \text { de Hogares } \\
\text { unipersonales } \\
\text { mayores de } 64 \text { años }\end{array}$ & 19,1 & 24,3 & 14,6 & 23,15 & 3,05 & 23,5 \\
\hline $\begin{array}{l}\text { Presión } \\
\text { migratoria* }(\%) \\
\end{array}$ & 6,8 & 2,84 & 5,10 & 3,58 & 2,92 & 0,07 \\
\hline $\begin{array}{l}\% \text { de población } \\
\text { sin estudios }\end{array}$ & 7,6 & 9,2 & 6,4 & 4,02 & 4,11 & 5,53 \\
\hline $\begin{array}{l}\text { Tasa de parol } \\
\text { desocupación }\end{array}$ & 22,8 & 24,8 & 21,7 & 3,12 & 3,68 & 3,97 \\
\hline $\begin{array}{l}\text { Renta per cápita } \\
\text { Por habitante }(2014) *\end{array}$ & 8.081 & 7.845 & 10.822 & s/d & $s / d$ & $s / d$ \\
\hline $\begin{array}{l}\text { Antigüedad de las } \\
\text { viviendas (en años) ** }\end{array}$ & 61,33 & 50,21 & 51,53 & s/d & $\mathrm{s} / \mathrm{d}$ & $\mathrm{s} / \mathrm{d}$ \\
\hline $\begin{array}{l}\text { Tamańo medio de las } \\
\text { viviendas } \\
\text { (m2) }\end{array}$ & 74,2 & 72,1 & 75,6 & $\begin{array}{c}64 \text { depto } \\
132 \\
\text { (PH/Casa) }\end{array}$ & $\begin{array}{c}76 \text { depto } \\
122 \\
\text { (PHCasa) }\end{array}$ & $\begin{array}{c}56 \text { depto } 101 \\
\text { (PH/Casa) }\end{array}$ \\
\hline $\begin{array}{l}\text { Precio delm2 } \\
\text { (EU Sev-USD LP) }^{* *}\end{array}$ & 1.066 & 1.062 & 2.207 & $\begin{array}{c}1.215 \text { depto } \\
912 \\
\text { (PH/Casa) }\end{array}$ & $\begin{array}{l}753 \text { depto } \\
704 \\
(\mathrm{PH} / \text { Casa) }\end{array}$ & $\begin{array}{l}1527 \text { depto } \\
988 \\
(\mathrm{PH} / \mathrm{Casa})\end{array}$ \\
\hline
\end{tabular}

Fuente: elaboración propia a partir de los Censos de Población (2011,

2010), registros catastrales (2019) y portales inmobiliarios (2020).

\section{Conclusiones. Estructura urbana y PATrones de SEgregación de los adultos MAYORES. ACERCA DE LAS ÁREAS DE VULNERABILIDAD URBANA}

Tanto Sevilla como La Plata, más allá de sus contextos regionales y nacionales diferentes, experimentan procesos similares en relación con la estructura urbana, las problemáticas de vulnerabilidad social y los patrones de segregación de los grupos envejecidos; lo mismo ocurre con las dinámicas de crecimiento urbano y transformación socio territorial. El análisis de las variables sintomáticas acerca de las condiciones socio habitacionales de las barriadas de estudio lleva a la conclusión final de que las áreas con mayor nivel de vulnerabilidad urbana no siempre se corresponden con las de mayor concentración de población envejecida; dicho de otra manera, los barrios más envejecidos no tienen por qué ser los que presenten en mayor medida situaciones de desventaja social. Por tanto, no siempre hay una relación conexa entre los dos fenómenos de estudio. De hecho, en Sevilla, en la actualidad, las zonas con más vulnerabilidad socio habitacional están habitadas por colectivos de edad joven-adulto, como por ejemplo el barrio de Los Pajaritos en el distrito Cerro-Amate o Las Vegas en el Polígono Sur (Ayuntamiento de Sevilla, 2011). Coincidimos con antecedentes de investigación que explican esta situación para la ciudad de Córdoba, donde los adultos mayores tienen una mejor situación socioeconómica que los jóvenes por ser sobrevivientes de un estado de bienestar (Peláez, 2006). En efecto, para el caso de La Plata se observa que las zonas con mejores condiciones 
de vida, como el casco fundacional y la localidad de Tolosa, son las que concentran la mayor presencia de adultos mayores.

Si bien lo anterior es cierto, hay dos tipologías de vulnerabilidad urbana que observan puntos de vinculación con los procesos del envejecimiento. Por una parte, la referente a tipologías de adultos mayores con mejor nivel socioeconómico pero emplazados en áreas urbanas, principalmente centrales, que están o han estado sujetas a procesos de renovación y/o regeneración y valorización. Las transformaciones socio territoriales en dichas áreas implican nuevas dinámicas comerciales, pérdida de identidad barrial y amenaza a la permanencia de los equipamientos sociales de uso comunitario, que pueden ser especialmente importantes para esta población. Por otro lado, con menor peso numérico y visibilidad en los mapas, está el envejecimiento demográfico como agravante de la situación de vulnerabilidad social asociada a las condiciones de vida en las áreas marginadas. En estos casos, las privaciones en cuanto a dotaciones de servicios de salud, accesibilidad y segregación se ven potenciadas.

En el primer grupo, el componente de vulnerabilidad se encuentra mayormente asociado a la propia condición de envejecimiento, que hace que sea más difícil adaptarse tanto a los cambios en el entorno urbano, como a su valorización. Aquí inciden en mayor medida las trayectorias del territorio y el ciclo de vida de los barrios. En el segundo caso, el envejecimiento constituye una dimensión secundaria que agrava el cuadro de privación material y desventaja socio económica y habitacional. Las iniciativas públicas orientadas al tratamiento del envejecimiento poblacional en las ciudades deben considerar esta diferencia.

\section{ReFERENCIAS}

Acosta, L. D. (2019). Factores asociados a la satisfacción vital en una muestra representativa de personas mayores de Argentina. Hacia Promoc. Salud. 24(1), 56-69. Doi: https://10.17151/hpsal.2019.24.1.6

Abades Porcel, M., y Rayón Valpuesta, E. (2012). El envejecimiento en España: ¿un reto o problema social? Gerokomos, 23(4), 151-155.

Alguacil, J., Camacho, J., y Hernández, A. (2014). La vulnerabilidad urbana en España. Identificación y evolución de los barrios vulnerables. EMPIRIA. Revista de Metodologia de Ciencias Sociales, 27, 73-94.

Ayuntamiento de Sevilla (2011). Diagnóstico de territorios desfavorecidos en la ciudad de Sevilla. Sevilla.

Casado, M. (2000). Los efectos del envejecimiento demográfico sobre el gasto sanitario: mitos y realidades. Gaceta Sanitaria, 15(2), 154-163.

CEPAL (2002). Vulnerabilidad sociodemográfica: Viejos y nuevos riesgos para comunidades, hogares y personas. Santiago de Chile: CEPAL, LC/R.

Coq Huelva, D. (2012). Crecimiento suburbano difuso y sin fin en el área metropolitana de Sevilla entre 1980 y 2010. Algunos elementos explicativos. Scripta Nova, XVI (397). Recuperado de http://www.ub.edu/geocrit/sn/sn-3 97.htm

Díaz Parra, I. (2009). Procesos de gentrificación en Sevilla en la coyuntura reciente. Análisis comparado de tres sectores históricos: San Luis-Alameda, Triana y San Bernardo (2000- 2006). Scripta Nova, XIII(304). Recuperado de h ttp://www.ub.edu/geocrit/sn/sn-304.htm

Egea Jiménez, C., y Nieto Calmaestra, J. A. (2015). Quién vive dónde: Las condiciones de habitabilidad de la población que vive en las grandes ciudades andaluzas. Granada: Editorial Universidad de Granada. Recuperado de https:/ /dialnet.unirioja.es/servlet/articulo?codigo $=6336536$

Frediani, J. (2009). Las Nuevas Periferias en el Proceso de Expansión Urbana. El Caso del Partido de La Plata. Geograficando. Revista de Estudios Geográficos, 5(5)103-125. Recuperado de http://www.memoria.fahce.unlp.e du.ar/art_revistas/pr.4445/pr.4445.pdf

Kaztman, R. (1999). Marco conceptual sobre activos, vulnerabilidad y estructuras de oportunidades. Montevideo, Uruguay: CEPAL- UN 
Laterza Calosso, J. (2015). Los espacios para la socialización y/o privacidad de personas mayores, en las residencias de larga estancia de Mar del Plata. Investigación + Acción, 18(17), 85-110.

Matteucci, L. G. (2015). Dime cuánto vale y te diré quién vive: Análisis de la relación entre los precios del suelo y el nivel socio-económico de los hogares en el partido de La Plata entre 2001 y 2010. [En línea] (Tesis de grado). Universidad Nacional de La Plata. Facultad de Humanidades y Ciencias de la Educación, La Plata, Buenos Aires, Argentina.

Moser, C. (1998). Reassessing urban poverty reduction strategies: The asset vulnerability framework. World Development. Washington, The World Bank, 1(26), 1-19.

Osorio Pérez, O. (2017). Vulnerabilidad y vejez: implicaciones y orientaciones epistémicas del concepto de vulnerabilidad. Intersticios sociales [online], 13, 1-34.

Peláez, E. (2006). Selectividad residencial de los adultos mayores en la ciudad de Córdoba. Astrolabio, 3 Recuperado de https://revistas.unc.edu.ar/index.php/astrolabio/article/view/199

Peláez, E. (2004). Características sociodemográficas del envejecimiento poblacional en la Provincia de Córdoba. En VI Jornadas Argentinas de Estudios de Población. Buenos Aires

Peláez, E., y Molinatti, F. (2016). Evolución de la segregación residencial y condiciones de habitabilidad de las viviendas de los adultos mayores en la ciudad de Córdoba, Argentina. Revista Márgenes, 13(19), 7-20.

Pérez Díaz, J. (1998), La demografía y el envejecimiento de las poblaciones. En A. S. Staab y L. C. Hodges (comp.), Enfermeria Gerontológica (pp. 451-463). México: McGraw Hill. Recuperado de http://digital.csic.es/bitstream /10261/14062/1/Staab98.pdf

Pérez Días, J., y Abellán García (2018). Envejecimiento demográfico y Vejez en España. Panorama Social, 18, 11-47. Recuperado de https://dialnet.unirioja.es/servlet/autor?codigo $=318498$

Prieto, M. B., y Formiga, N. (2008). Envejecimiento y vulnerabilidad sociodemográfica. El caso de Babia Blanca Argentina. Ponencia presentada en III Congreso de la Asociación Latinoamericana de Población, Córdoba, Argentina, del 24 al 26 de septiembre. http://www.alapop.org/alap/images/DOCSFINAIS_PDF/ALAP_200 8_FINAL_296.pdf

Relli Ugartamendía, M. (2018). Política de regularización del hábitat popular urbano: provincia de Buenos Aires y partido de La Plata, 1983-2015 (Tesis de doctorado en Geografía), UNLP. Publicada en https://doi.org/10.3 $5537 / 10915 / 70079$

Rodríguez Vignoli, J. (2000). Vulnerabilidad demográfica: una faceta de las desventajas sociales. Santiago: CEPAL, Serie Población y Desarrollo No 5. Recuperado de https://red- ahdiversur.weebly.com/ uploads/3/1/2/1/31216499/vulnerabilidad_rodriguez_vignoli.pdf

Rodríguez Rodríguez. V. (2018). El marco de las políticas de vejez en Europa. Panorama Social, 28, 147-160. Recuperado de https://encage-cm.es/wp- content/uploads/2016/12/Rodriguez-2018-el-marco-delas-politicas-de-vejez-en-eur.pdf

Sánchez González, D. (2007). Envejecimiento demográfico urbano y sus repercusiones socio-espaciales en México. Retos de la planeación gerontológica. Revista Norte Grande, 38, 45-61. Recuperado de https://scielo.conicyt.cl $/$ scielo.php?script $=$ sci_arttext\&pid=S0718-34022007000200003

Saraví, G. (Ed.) (2006). De la pobreza a la exclusión: continuidades y rupturas de la cuestión social en América Latina. Buenos Aires: Prometeo.

Sugranyes, A., y Mathivet, C. (edts) (2010). Ciudades para tod@s. Por el derecho a la ciudad, propuestas y experiencias. Santiago de Chile: Habitat International Coalition (HIC). Recuperado de http://www.hic-gs.org/content/Cu idades\%20para\%20todos\%20HIC-2011.pdf

Tomas, M. S. (2007). Envejecimiento y feminización de la población de la Provincia de Buenos Aires: dimensiones, perspectivas e interrogantes. Ponencia presentada en las IX Jornadas Argentinas de Estudio de Población, red AEPA, 31 de octubre al 2 de noviembre, en Huerta Grande, Córdoba.

Valle Ramos, C. del (2007). Envejecimiento demográfico y migraciones en la ciudad de Sevilla. Papeles de Geografía, 45-46, 187-201. Recuperado de https://revistas.um.es/geografia/article/view/43371/41631 
Valle Ramos, C del., Egea Jiménez, C., y Nieto Calmaestra, J. A. (2020). Urban renewal processes as mitigators of disadvantaged and vulnerable situations: analysis in Seville city. Boletin de la Asociación de Geógrafos Españoles, 87. https://doi.org/10.21138/bage.2981

Vallejo, G. (2000). De los Apeninos a La Plata. Los italianos en la construcción de la "nueva capital". Anuario del Instituto de Historia Argentina, 1, 153-173. Recuperado de http://www.memoria.fahce.unlp.edu.ar/art_revista s/pr.2914/pr.2914.pdf

\section{Notas}

1 De acuerdo con la metodología censal adoptada por el INDEC, se consideran hogares con NBI a aquellos que presentan al menos una de las siguientes características: vivienda inconveniente, Carencias sanitarias, Condiciones de Hacinamiento, Inasistencia escolar, Capacidad de subsistencia Según los datos, los componentes de mayor incidencia son el Hacinamiento y el déficit de vivienda: La Plata, 2,23 y 6,01\%; Tolosa, 0,24 y 0,26\% y Monasterios 0,79 y 0,64\%, respectivamente. 